\title{
Dengue Vaccine Controversy Awareness, Vaccine Health Literacy, and Vaccine Acceptability among Mothers in Select Rural Communities
}

\author{
https://doi.org/10.37719/jhcs.2020.v2i2.oa005
}

EARL FRANCIS R. SUMILE, Ph.D., RN https://orcid.org/0000-0002-7754-5944

JOHN HOWELL E. DIRIC, BSN, RN

ZARIAH MONICA A. DORADO, BSN, RN
KATE T. DUMAUA, BSN, RN

MONICA JULIA RYVEN D. ECURA, BSN, RN JOSE MARIA D. DUMAYA

College of Nursing, University of Santo Tomas, Manila, Philippines

Corresponding author's email: nurse.earlfrancisrsumile@gmail.com

\section{Abstract}

Background: Immunization is a vital public health service that reduces the transmission of communicable diseases among population groups. In the Philippines, reports of adverse reactions from the dengue vaccine raised doubts about its safety and efficacy. This in turn can bring about a decline in vaccine acceptability among mothers and later on result in reduced vaccine coverage in the country.

Methods: Descriptive cross-sectional correlational design was used to explicate the influence of dengue vaccine controversy awareness and vaccine health literacy on vaccine acceptability among mothers. Data were collected among 200 mothers from August - November 2018 in rural communities of Bulacan. Stepwise forward and backward multiple linear regression were done to determine the relationships between the variables of interest.

Results: The majority of the mothers who participated belong to the 26-35-year-old age bracket and received a basic education. Mothers' functional health literacy $(B=0.189, p=0.006)$ and critical health literacy $(B=0.247, p=0.000)$ revealed a significant relationship to vaccine acceptability. However, communicative health literacy $(B=0.008, p=0.917)$ showed no significant relationship to vaccine acceptability. Moreover, the dengue vaccine controversy awareness $(B=0.057, p=0.415)$ had no 
significant relationship with the mothers' vaccine acceptability.

Conclusion: Health literacy favorably influences vaccine acceptability. Likewise, awareness of controversies surrounding dengue vaccination is not a deterrent to the decision of mothers to continue submitting their children to immunization. Hence, nurses should invest more in educational interventions to promote compliance of communities to the national immunization program and engage stakeholders to support its implementation.

Keywords: Health Literacy, Vaccination, Preventive Health Services, Vaccination Coverage, Public Health

\section{Introduction}

\section{Background}

- he Philippines as a developing nation has long been struggling with communicable diseases. To help reduce the spread of these conditions particularly among children, the Philippine government launched the Expanded Program on Immunization in 1976 (Department of Health, 2020a). Employing mandatory vaccination of children as the key strategy, the program was aimed at developing herd immunity in the population, thereby preventing unnecessary deaths from common childhood infections. Initially, six vaccines were intended for the prevention of diseases such as tuberculosis, diphtheria, pertussis, tetanus, measles, and poliomyelitis (DOH, 2020). Over the years, the list of biologicals has grown to address the changing epidemiological situation of the country. In 2011, Republic Act 10152 or the Mandatory and Basic Immunization Act was implemented to intensify the public health campaign of protecting vulnerable population groups from various infectious diseases $(\mathrm{DOH}, 2020)$. To date, this law is considered as the legal basis of the immunization program in the Philippines and includes vaccines against pneumonia, meningitis, measles, mumps, and rubella. With the successful implementation of the immunization program, the Philippines has significantly reduced the under-five mortality almost meeting the target set in the Millennium Development Goals of the United Nations. Based on the United Nations Children's Fund (UNICEF) 2020 database, the Philippines ' under-five mortality rates has improved from 37.7 per 1000 live births in 2000 to 27.3 last 2019.

In 2016, the Department of Health launched a special mass immunization for children 9 to 14 years of age against dengue hemorrhagic fever. Areas covered were limited to Metro Manila, Central Luzon, and Southern Luzon, as these are the regions with the highest number of reported cases of dengue in the country. However, reports of adverse reactions from the vaccine raised speculations and doubts about its safety and efficacy that led to vaccine hesitancy (DOH, 2018). According to McClure et al. (2017), vaccine hesitancy is demonstrated by increased requests for alternative vaccination schedules or by altogether postponing or declining vaccines Consequently, 
the drop in vaccine coverage in the country is attributed by health authorities to this controversy. Based on the 2018 report of the Department of Health, the percentage of fully immunized children in the country is at $66 \%$, compared to $75 \%$ in 2014 .

Nurses account for the majority of the human resource for health in the country. As providers of primary care in communities, public health nurses are in a pivotal stance to mainstream health promotion and health protection activities. The national immunization program (also referred to as Expanded Program on Immunization) significantly reduces the risk of acquiring communicable diseases among children and mothers. To achieve its aim, health literacy and vaccine acceptability are prerequisites. Thus, understanding whether current issues in the country pose negative influences on parents' decisions to submit their children for immunization is paramount. The ability to process and utilize information to improve and maintain one's health is termed as health literacy. The World Health Organization (2016), cites that health literacy of the population is essential to effectively implement public health programs. In a country where the health literacy of the population is a challenge, it is critical for health professionals like nurses to communicate information on the value of immunization. Hence, nurses in public health can better engage communities to avail of various healthcare services to increase their wellness and ultimately achieve better health outcomes.

\section{Aims/Objectives}

In line with the situations discussed above, this study investigated the relationship of vaccine health literacy and denaue vaccine controversv awareness with vaccine accentabilitv (Figure 1).

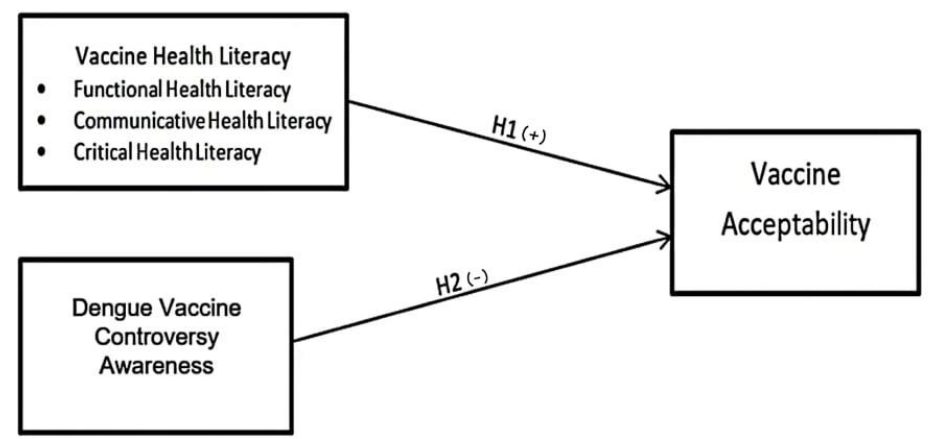

Figure 1. Proposed Model

\section{Methods}

\section{Study Design and Setting}

A descriptive cross-sectional and correlational design was used to explicate the relationships of dengue vaccine controversy awareness and vaccine health literacy to the vaccine acceptability among mothers. 
Being a study in the area of public health, fieldwork was carried out by the researchers in the province of Bulacan. The site was chosen for two main reasons. First, Bulacan is part of Central Luzon which was included in the special mass immunization for dengue last 2016. Second, the proximity of the province to Metro Manila permits free movement of people, goods, technology, and information across borders. Communities specifically from the municipalities of Plaridel and Marilao were chosen due to the existence of community people's organizations in the area that spearhead various health-related activities.

\section{Sampling Technique}

Consecutive sampling was utilized among the mothers who visited the rural health unit or health center and met the following inclusion criteria: (1) able to read and write (2) and with children who are six months to five years of age or with children who have been vaccinated by the government under the immunization program of the Philippines. Meanwhile, the following are the exclusion criteria: (1) vulnerable individuals such as pregnant women, minors, prisoners, those with diminished mental capacity, and older adults, (2) those whose children have been included in the dengue special immunization activity, and (3) mothers whose children did not receive any form of vaccination. Consecutive sampling is highly useful when there is limited availability of subjects or when using stringent selection criteria that may threaten the generalizability of the results. A total of 200 mothers consented to participate in the study and sampling adequacy was assessed using post hoc power analysis, reflecting an effect size of 0.093 and an observed statistical power of 0.929 .

\section{Research Instruments}

Three tools were deployed to measure the variables of interest namely: vaccine acceptability, vaccine health literacy, and dengue vaccine controversy awareness. All instruments were subjected to face and content validity by experts and were pilot tested for reliability testing. Likewise, permission from the primary tool developers was sought. With their consent, the instruments originally devised in English were translated to Filipino. Language experts assisted in accomplishing the translation. The researcher-made Dengue Vaccine Controversy Awareness tool has two-parts: subject demographic profile and questions about the vaccine controversy. The questionnaire is composed of 7 items derived from extant literature with dichotomous options of "yes or no". The tool was pilot tested twice and then modified until it yielded a reliability score of 0.611 which is considered acceptable for newly developed instruments (Taber, 2016). The Vaccine Health Literacy Scale is the second tool used in this study and was adopted from the work of Aharon and colleagues (2016). This instrument is specifically intended to measure the health literacy of mothers regarding vaccines in three domains (functional, critical, and communicative), with 14 items ranked on a Likert scale ranging from $1=$ "not at all" to $4=$ "very much so". This tool also has good internal consistency (Cronbach's alpha=0.84). The last instrument is the Vaccine Acceptability Scale (Sarathchandra et al., 2018), which was used to determine the parents' acceptability of vaccines. It measures the key facets of vaccine acceptance through agreement or disagreement in five areas 
namely: perceived safety of vaccines; perceived effectiveness and necessity of vaccines; acceptance of the selection and scheduling of vaccines; positive values and affect toward vaccines; and perceived legitimacy of authorities to require vaccinations. Face validity was determined through three experts and reliability testing showed a result of 0.734 . Finally, to ensure the appropriateness of the tools to the subjects, factors such as reading level, length of time, cultural differences, beliefs, and language barriers were all considered by the researchers.

\section{Data Gathering Procedure and Data Analysis}

After securing technical and ethical clearance, the researchers made initial contact with prospective communities. Permission from local government authorities was also sought before the actual visit to the sites. A community assembly was then scheduled to meet prospective participants by the community organization based on the established eligibility criteria. However, with a low turnout during the assembly, the researchers conducted a series of home visits after a prior notice was given through local leaders of the communities. On the course of the said home visit, researchers were accommodated in the common area of the household without any other members of their family present. Informed consent was secured after explaining the details of the protocol. Questionnaires were then handed over and were accomplished by the subjects within an average of 30 to 45 minutes. Token of appreciation was provided to the participants at the end of each visit consisting of grocery items to compensate for their shared time and information.

The study utilized the Statistical Package for Social Sciences (SPSS) to perform descriptive statistics to measure the mean and frequency and percentage of continuous and categorical data. Standard deviation (SD) was used to assess the variability of scores. Finally, stepwise forward and backward multiple linear regression was done to determine the relationships among the variables of interest.

\section{Ethical Considerations}

The study was conducted with the utmost consideration for the rights of research participants and adherence to the National Ethical Guidelines of Health Research. In particular, the principles of beneficence, justice, informed consent, data privacy, and confidentiality, as well as ensuring social value, and protection of vulnerable populations were observed throughout the conduct of this investigation. Clearance from the researchers' institutional ethics review committee (Protocol Number: USTCON-2018 SR05) was obtained before commencing any fieldwork. Free and prior informed consent was obtained. Subjects are briefed on the nature, purpose, risks/benefits of the study, and that participation is purely voluntary. To ensure the privacy of the family, a household visit was conducted in their receiving area without any other members present. Forms accomplished by subjects were coded and anonymized to maintain confidentiality. Likewise, all forms containing information collected from the participants of the study were secured in a locked filing cabinet. 


\section{Result}

The demographic profile of the respondents (Table 1) revealed that the majority of the mothers $(99.5 \%)$ received a basic education, which includes primary and secondary levels. In terms of age, most of the respondents were from the 26-35-year-old age bracket.

Table 1. Demographic Profile $(n=200)$

\begin{tabular}{ccc}
\hline & Frequency & Percent \\
\hline Age & & \\
$\leq 20$ & 14.0 & $7.0 \%$ \\
$21-25$ & 35.0 & $17.5 \%$ \\
$26-30$ & 37.0 & $18.5 \%$ \\
$31-35$ & 42.0 & $21.0 \%$ \\
$36-40$ & 30.0 & $15.0 \%$ \\
$41-45$ & 24.0 & $12.0 \%$ \\
$46-50$ & 9.0 & $4.5 \%$ \\
$51-55$ & 2.0 & $1.0 \%$ \\
$56-60$ & 7.0 & $3.5 \%$ \\
Educational Attainment & & \\
No formal Education & 1 & $0.5 \%$ \\
Elementary Level & 40 & $20.0 \%$ \\
Elementary Graduate & 7 & $3.5 \%$ \\
High School Level & 70 & $35.0 \%$ \\
High School Graduate & 50 & $25.0 \%$ \\
College Level & 22 & $11.0 \%$ \\
College Graduate & 10 & $5.0 \%$ \\
\hline
\end{tabular}

The level of the mother's awareness of the Dengue vaccine controversy is high with an average score of 4.37 out of 7 questions in the tool. Vaccine health literacy was measured in 3 areas, such as functional, communicative, and critical domains, which reported high mean scores. Aharon (2016) states that a score approximating a value of 4 is interpreted as a high degree of health literacy and a score approximating a value of 1 is interpreted as a low degree of health literacy. Table 2 presents the mean scores for awareness, vaccine health literacy, and vaccine acceptability of the subjects in this study.

Table 2. Mean of Dengue Vaccine Controversy Awareness, Vaccine Health Literacy, and Vaccine Acceptability

\begin{tabular}{lcc}
\hline & Mean & Standard Deviation \\
\hline $\begin{array}{l}\text { Dengue Vaccine Controversy } \\
\text { Awareness }\end{array}$ & 4.37 & 2.028
\end{tabular}




\begin{tabular}{lcc}
\hline & Mean & Standard Deviation \\
\hline Vaccine Health Literacy & 3.23 & 0.588 \\
Functional Health Literacy & 3.14 & 0.757 \\
Communicative Health Literacy & 2.95 & 0.873 \\
Critical Health Literacy & 3.65 & 0.735 \\
Vaccine Acceptability & 5.28 & 0.896 \\
\hline
\end{tabular}

Respondents' functional health literacy $(B=0.189 ; p=0.006)$ and the critical health literacy $(B=0.247 ; p=0.000)$ showed significant relationships with vaccine acceptability (Table 3$)$. Meanwhile, communicative health literacy $(B=0.008 ; p=0.917)$ had no significant relationship with the vaccine acceptability of the mothers. However, although two categories of health literacy showed a significant relationship on vaccine acceptability, the score for the overall health literacy $(B=0.024 ; p=0.789)$ showed no significance on vaccine acceptability of the mothers. For the associations between dengue vaccine controversy awareness and vaccine acceptability, no significant relationship $(B=0.057$; $\mathrm{p}=0.415)$ was determined statistically (Table 4).

Table 3. Stepwise Linear Regression of Vaccine Health Literacy to the Mother's Vaccine Acceptability

\begin{tabular}{lcccl}
\hline & Std. Error & Beta & P-Value & Interpretation \\
\hline Functional HL & 0.069 & 0.189 & 0.006 & Significant \\
Critical HL & 0.069 & 0.247 & 0.000 & Significant \\
Communicative HL & 0.051 & 0.008 & 0.917 & Not Significant \\
Overall Vaccine HL & 0.047 & -0.024 & 0.789 & Not significant \\
\hline
\end{tabular}

Table 4. Correlation of Dengue Vaccine Controversy Awareness to Vaccine Acceptability

\begin{tabular}{lcccc} 
& Std. Error & Beta & P-Value & Interpretation \\
\hline $\begin{array}{l}\text { Dengue Vaccine } \\
\text { Controversy Awareness }\end{array}$ & .057 & .415 & Not Significant \\
\hline
\end{tabular}

Finally, using stepwise regression, the emerging model that reflects the influences of awareness of the dengue vaccine controversy, and health literacy on vaccine acceptability of mothers is shown in Figure 2.

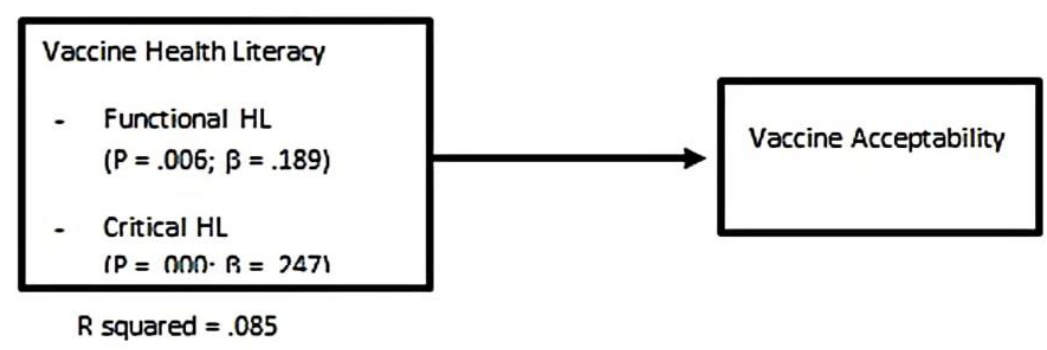

Figure 2. Emergent Model 


\section{Discussion}

Vaccine health literacy is viewed as the people's attitude and belief towards vaccination to understand the main determinants of vaccine acceptability (Yousif et al., 2013). There are three levels of health literacy in general: functional, communicative, and critical. Functional health literacy is the basic ability of an individual to read and write. Communicative health literacy is the ability of an individual to process health information and know its significance. Lastly, critical health literacy refers to the ability of a person to analyze health information (Aharon et al., 2016). Vaccine information is considered to be complex which is why comprehending information about vaccines requires a certain level of health literacy and skills. This could be a challenge for people who have low health literacy (Lorini, 2018). In this study, the mothers who participated all reported high scores in these areas. This finding may seem odd since most studies showed that higher educational attainment is linked to higher health literacy and ultimately to better health outcomes (Chabra et al., 2015). Also, a higher educational level results in a better understanding of health information among parents than those with lower educational attainment (Yousif et al., 2013). However, the findings of this study state otherwise. The majority of the mothers received only basic education (primary and secondary levels) but they recorded high scores for their vaccine health literacy. At the minimum, individuals at this level are expected to demonstrate the ability to read, write, and comprehend health information. In contrast, Rowlands (2014) suggests that health literacy is a complex concept, ranging from basic skills in reading and numeracy to advanced cognitive skills needed to critically analyze information. Factors that account for this finding may include the respondents' prior experiences of availing healthcare services and access to health information from multiple sources like social media. Information directed to the parents about vaccination against disease, their access to modern mass media, and their socio-cultural behaviors can be major determinants of vaccination compliance (Phimmasane et al., 2010).

Vaccine acceptability of mothers who participated in this study showed significant relationships with functional and critical health literacy. However, the overall health literacy score did not. This gap may be related to how the subjects accomplished the tool due to the time required to finish it or possibly the items for them are already redundant. Still, it is noteworthy to point that in this study, vaccine health literacy positively influences the mothers' acceptability towards vaccines. This means that they have a sufficient understanding of the value of vaccines and that they will utilize this preventive service for the benefit of their children's health.

Immunization is an essential public health service that has prevented common childhood diseases in the country. Over the years, it has been implemented by the Department of Health through local government units as a major contributor to the achievement of reducing under-five child mortality. The cooperation of parents rooted in their confidence in the efficacy and safety of vaccines account for its wide acceptability in communities. However, in the presence of controversies surrounding new vaccines, the risk of non-compliance to immunization programs becomes eminent. 
This problem is further exacerbated if parents have a limited understanding of vaccines as reported by Pugliese-Garcia et al. (2018). In 2018, the Philippine Pediatric Society noted an increasing vaccine refusal in the country brought about by the dengue vaccine controversy due to the reported adverse effects attributed to it. As a consequence, there was a marked decline in parents getting vaccinations for their children for other diseases such as pneumonia, measles, polio, and tuberculosis (CNN Philippines, 2018). According to Dube et al. (2015), the adverse events that can result from the vaccines can affect parents' vaccine decisions in general and newly created vaccines usually cause more hesitancy towards vaccination. As such, implementing new vaccines should be coupled with the provision of information tailored for the individual and the vaccine it concerns. (Eilers et al., 2017)

In this study, the mothers demonstrated a high level of awareness of the dengue vaccine controversy. Surprisingly, their awareness is not significantly related to their vaccine acceptability. This is a unique case that runs contrary to most studies highlighting the negative consequence of issues on new vaccines to immunization utility. In other words, the mothers regard the value of immunization as an essential health service that improves the wellness of their children. On one hand, acceptance of vaccination is an outcome behavior resulting from a complex decision-making process that can be potentially influenced by a wide range of factors. Vaccine hesitancy, on the other hand, is complex and context-specific, varying across time, place, and vaccines, and includes factors such as complacency, convenience, and confidence (WHO, 2014). Further, the level of the parent's education, occupation, number of children, and age of the children are contributing factors in attending immunization services and immunization status of children (Phimmasane et al., 2010). Indeed, previous experiences with vaccination reinforced the respondents' understanding of the benefit of this health technology. Thus, it can be considered as a key element to explain this unique phenomenon. Valido et. al (2018) stated that among parents, vaccine acceptability commonly results from prior experience with the immunization program and the communication they received from health authorities. Also, the massive information drive promoting the effectiveness of immunization undertaken by the government in response to the controversy contributed to this finding. This echoes the work of Smith et al. (2017), which highlighted the value of communicating information to parents to promote increased vaccine uptake.

\section{Conclusion}

The ability to understand, process, and utilize health information supports the acceptance of health services. Such is the outcome of this study that reinforces that health literacy promotes vaccine acceptability of mothers. Awareness of controversies surrounding dengue vaccination is not a deterrent to the decision of mothers to continue submitting their children to the national immunization program. This creates a perfect opportunity for nurses and other healthcare providers to invest in educational interventions that will foster positive health-seeking behaviors among mothers and other population groups. Likewise, it is essential to utilize multiple platforms for disseminating correct health information to communities. Considering the acceptability of vaccines, nurses across 
all settings should engage stakeholders and decision-makers in government. Nurses should step forward in communicating evidence that will support better funding for immunization services in communities and thereby contribute to better health outcomes. With limitations encountered in this research, future studies are recommended to look into other factors that influence vaccine acceptability on a wider population.

\section{Conflict of Interest}

The authors have no conflict of interest to disclose.

\section{Acknowledgment}

The authors would like to extend their gratitude to the communities who made this study possible and experts for their assistance in the completion of this research. In particular, the authors would like to thank Dr. Gian Carlo Torres, Asst. Prof. Laurence Lloyd Parial, and Mr. John Rey Macindo RN for their support.

\section{Funding}

The authors have no funding to disclose.

\section{Author Contribution}

Earl Francis Sumile: conception and design of the work; analysis and interpretation of data, drafting and critical revision of the paper and the article, oversaw final approval of the study; and accepts accountability on all aspects of the work. John Howell Diric: acquisition of data and analysis and interpretation, approving the final study, accountability on all aspects of the work. Zariah Monica Dorado: acquisition of data and analysis and interpretation, approving the final study, accountability on all aspects of the work. Kate Dumaua: acquisition of data and analysis and interpretation, approving the final study, accountability on all aspects of the work. Monica Julia Ryen Ecura: acquisition of data and analysis and interpretation, approving the final study, accountability on all aspects of the work. Jose Maria Dumaya: conception and design of the work, including the acquisition of data; analysis and interpretation of data, approving the final study, and accountability on all aspects of the work. 


\section{References}

Aharon, A., Nehama, H., Rishpon, S. \& Baron-Epel, O. (2016). Parents with high levels of communicative and critical health literacy. Patient Education and Counseling.100(4), 768-775. https://doi.org/10.1016/j.pec.2016.11.016

Chhabra, R., Chisolm, D., Bayldon, B., Quadri, M., Sharif, I., Velasquez, J., ENcalada, K., Rivera, A., Harris, M., Levites-Agababa, E., \& Yin, H. (2018). Evaluation of Pediatric Human Papillomavirus Vaccination Provider Counseling Written Materials: A Health Literacy Perspective. Academic Pediatrics, 18(2), S28-S36. https://doi.org/10.1016/j.acap.2017.08.004

CNN Philippines. (2018). Doctors: Parents Refusing Vaccines Due to Dengvaxia Scare. https://cnnphilippines.com/news/2018/02/03/doctors-parents-refusing-vaccines-dengvaxia.html.

Department of Health Philippines. (2020a). Expanded Program on Immunization. https://www.doh.gov.ph/expanded-program-on-immunization

Department of Health Philippines. (2018). DOH Identifies Vaccine Hesitancy as one of the reasons for a measles outbreak. https://www.doh.gov.ph/node/16721.

Dubé, E., Laberge, C., Guay, M., Bramadat, P., Roy, R., \& Bettinger, J. (2013). Vaccine hesitancy: an overview. Human vaccines \& immunotherapeutics, 9(8), 1763-1773. https://doi.org/10.4161/hv.24657

Eilers, R., de Melker, H. E., Veldwijk, J., \& Krabbe, P. (2017). Vaccine preferences and acceptance of older adults. Vaccine, 35(21), 2823-2830. https://doi.org/10.1016/j.vaccine.2017.04.014

Lorini, C., Santomauro, F., Donzellini, M., Capecchi, L., Bechini, A., Boccalini, S., Bonanni, P., \& Bonaccorsi, G. (2018). Health literacy and vaccination: A systematic review. Human vaccines \& immunotherapeutics, 14(2), 478-488. https://doi.org/10.1080/21645515.2017.1392423

McClure, C. C., Cataldi, J. R., \& O'Leary, S. T. (2017). Vaccine Hesitancy: Where We Are and Where We Are Going. Clinical Therapeutics, 39(8), 1550-1562. https://doi.org/10.1016/j.clinthera.2017.07.003

Phimmasane, M., Douangmala, S., Koffi, P., Reinharz, D., \& Buisson, Y. (2010). Factors affecting compliance with measles vaccination in Lao PDR. Vaccine, 28(41), 6723-6729. https://doi.org/10.1016/j.vaccine.2010.07.077

Pugliese-Garcia, M., Heyerdahl, L. W., Mwamba, C., Nkwemu, S., Chilengi, R., Demolis, R., Guillermet, E., \& Sharma, A. (2018). Factors influencing vaccine acceptance and hesitancy in three informal settlements in Lusaka, Zambia. Vaccine, 36(37), 5617-5624. https://doi.org/10.1016/j.vaccine.2018.07.042

Rowlands, G. (2014). Health literacy. Human Vaccines \& Immunotherapeutics, 10(7), 2130-2135. https://doi.org/10.4161/hv.29603

Sarathchandra, D., Navin, M., Largent, M., McCright, A. (2018). A survey instrument for measuring vaccine acceptance. Preventive Medicine, 109, 1-7. https://doi.org/10.1016/j.ypmed.2018.01.006

Smith, L. E., Amlôt, R., Weinman, J., Yiend, J., \& Rubin, G. J. (2017). A systematic review of factors affecting vaccine uptake in young children. Vaccine, 35(45), 6059-6069. https://doi.org/10.1016/j.vaccine.2017.09.046

Taber, K. S. (2017). The Use of Cronbach's Alpha When Developing and Reporting Research Instruments in Science Education. Research in Science Education, 48(6), 1273-1296. https://doi.org/10.1007/s11165-016-9602-2

United Nations Children's Emergency Fund Philippines. (2020). Philippines (PHL) - Demographics, Health \& Infant Mortality. https://data.unicef.org/country/phl/. 
Valido, E. M., Laksanawati, I. S. \& Utarini, A. (2018). Acceptability of the dengue vaccination among parents in urban poor communities of Quezon City, Philippines before and after vaccine suspension. BMC Research Notes 11, 661. https://doi.org/10.1186/s13104-018-3766-y.

World Health Organization. (2014). Report of the SAGE Working Group on Vaccine Hesitancy. https://www.who.int/immunization/sage/meetings/2014/october/1_Report_WORKING_GROUP_vac cine_hesitancy_final.pdf

World Health Organization. (2016). The Mandate for Health Literacy. https://www.who.int/healthpromotion/conferences/9gchp/health-literacy/en/.

Yousif, M. A., Albarraq, A., Abdallah, M., Elbur, A. (2014). Parents' Knowledge and Attitudes on Childhood Immunization, Taif, Saudi Arabia. Journal of Vaccines \& Vaccination, 5(1). https://doi.org/10.4172/2157-7560.1000215

\section{About the Authors}

Earl Francis R. Sumile, PhD, RN, is a nurse by profession, an educator by practice, and a community development worker by heart. Obtained both his Bachelor of Science in Nursing and Master of Arts in Nursing from the University of Santo Tomas; and his Doctor of Philosophy in Nursing from the University of the Philippines Manila. Mentored nursing students both in lecture and clinicals for Community/Public Health Nursing over the last 16 years as Assistant Professor at the University of Santo Tomas until his retirement last August 2020. His program of research focuses on advancing health promotion of underserved population groups and advocating for people empowerment among communities.

John Howell E. Diric, BSN, RN, ranked 10th place in the November 2019 Nurse Licensure Exam and currently works in a specialized hospital in Metro Manila. He obtained his Bachelor of Science in Nursing from the University of Santo Tomas.

Zariah Monica A. Dorado, BSN, RN, is a graduate of the University of Santo Tomas for her Bachelor of Science in Nursing degree and currently working as a staff nurse in a tertiary hospital in Metro Manila.

Kate T. Dumaua, BSN, RN, is currently pursuing her Doctor of Medicine degree from the UST Faculty of Medicine and a graduate of the University of Santo Tomas for her Bachelor of Science in Nursing degree.

Monica Julia Ryven D. Ecura, BSN, RN, is a professional nurse currently working in a district hospital in the province of Zambales and obtained her Bachelor of Science in Nursing from the University of Santo Tomas.

Jose Maria D. Dumaya, is a professional nurse and a graduate of the University of Santo Tomas for his Bachelor of Science in Nursing degree. He is presently studying at the Far Eastern University - NRMF under the Doctor of Medicine program. 